\title{
Prediction of critical micelle concentration of cationic surfactants using connectivity indices
}

\author{
Anna Mozrzymas • Bożenna Różycka-Roszak
}

Received: 7 January 2010 / Accepted: 22 September 2010 / Published online: 7 October 2010 C The Author(s) 2010. This article is published with open access at Springerlink.com

\begin{abstract}
Relationship for predicting $\log c m c$ for cationic surfactants having chloride as counterion from only molecular connectivity indices was found. It is suggested that the index ${ }^{0} \chi^{v}$ includes some information about hydrophobicity while indices ${ }^{4} \chi_{p c}$ and ${ }^{4} \chi_{p c}^{v}$ include some information about hydrophilicity of the cationic surfactants studied. The structures of 23 compounds used for the correlation are quite diverse.
\end{abstract}

Keywords Cationic surfactants · Chloride - QSPR · cmc ·

Molecular connectivity indices

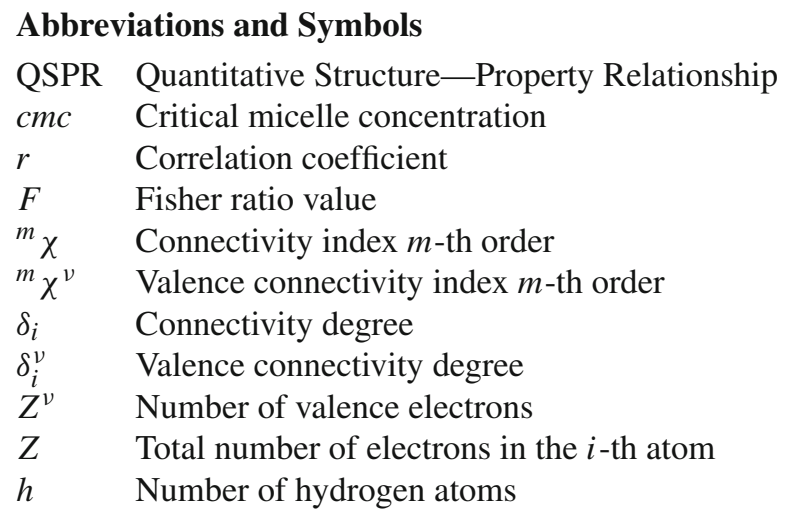

A. Mozrzymas $(\bowtie) \cdot$ B. Różycka-Roszak

Department of Physics and Biophysics, Wrocław University of Environmental and Life Sciences, ul.

Norwida 25, 50-375, Wrocław, Poland

e-mail: anna.mozrzymas@up.wroc.pl 


\section{Introduction}

Cationic surfactants are widely used in various fields of industry and biology because they show good bactericidal properties and they are innocuous for human beings and animals. Quaternary ammonium salts are typical cationic surfactants. Their utility results from their anti-electrostatic and anti-corrosion properties, and in particular from their anti-microbial properties with respect to a large spectrum of bacteria, algas and viruses.

The critical micelle concentration $(\mathrm{cmc})$ is the most useful parameter for characterizing surfactants. The $c m c$ is defined as the concentration at which molecules begin to aggregate to form micelles. During this process many important physicochemical properties such as surface tension, conductivity and detergency change significantly. These properties are important for many industrial and biological systems, therefore predicting the critical micelle concentration directly from the structure of a molecule by quantitative structure-property relationship (QSPR) analysis is of great interest. The first correlation was given by Klevens [1] who empirically found that logarithm of $\mathrm{cmc}$ linearly decreases with increasing length of an alkyl chain. Recently, the QSPR was used for predicting the $c m c$ values of nonionic surfactants [2-5], anionic surfactants [5-8] and cationic surfactants [9]. In papers [2,3,10] molecular connectivity indices together with another topological descriptors are correlated with $\mathrm{cmc}$ values.

Molecular connectivity indices have been widely used as molecular structural descriptors because they are rich in structural information. In our study we use ten indices: five connectivity indices and five valence connectivity indices, from zeroth to fourth order in both cases. These topological descriptors contain some information about the molecule. Kier and Hall [11] stated that ${ }^{0} \chi$ and ${ }^{0} \chi^{\nu}$ include information about atoms contained in a molecule; information about molecular volume and molecular surface area is encoded in the ${ }^{1} \chi$ and ${ }^{1} \chi^{\nu}$ indices, ${ }^{2} \chi$ indices carry an information about three-atom fragments which are the minimum number necessary to describe a plane; an information about the number of branch points in the molecule could be contained in ${ }^{3} \chi_{c}$; the indices ${ }^{4} \chi_{p c}$ and ${ }^{4} \chi_{p c}^{v}$ include information about the number of ring substituents, the length of the substituents and the heteroatom type of substituent. Therefore, these descriptors have been successfully used to correlate and to predict the physicochemical properties that depend on structure of the molecule.

In a previous paper [12] we derived the relationship between $\log c m c$ and molecular connectivity indices for nonionic surfactants. Using the set of descriptors listed above we have obtained the relationship that contains four indices: ${ }^{1} \chi,{ }^{1} \chi^{\nu},{ }^{4} \chi_{p c}^{\nu}$ and ${ }^{0} \chi$. The previous QSPR study shows that surfactants properties, especially the critical micelle concentration, can be predicted on the basis of molecular structure by using the molecular connectvity indices only. Continuing our work, we decided to correlate Log cmc of cationic surfactants with topological indices too. To the best of our knowledge it is for the first time. Admittedly, M. Jalali-Heravi and E. Konouz [9] also correlated $\log c m c$ of some quaternary ammonium salts with topological descriptors, but they used topological descriptors as well as the electronic parameters of the total energy of the molecule.

The critical micelle concentration depends not only on geometrical factors but also on a number of other parameters, among them a kind of counterion and electrostatic 
Table 1 Experimental values of $c m c$ of compounds study

\begin{tabular}{lll}
\hline Code & Name of Compound & $c m c(\mathrm{mM})[\mathrm{Ref}]$. \\
\hline CnTAC & $\begin{array}{l}N \text {-alkyl- } N, N, N \text {-trimethylammonium } \\
\text { chlorides }(n=10,12,14,16,18)\end{array}$ & $61[13], 21[14], 4.5[13], 1.4,0.35[15]$ \\
DDAC & $N$-dodecyl- $N, N$-dimethylammonium chloride & $15.8[16]$ \\
$\mathrm{C}_{\mathrm{n}} \mathrm{AC}$ & dodecylamine hydrochlorides $(n=8,10,12)$ & $200,48[1], 13.5[16]$ \\
$\mathrm{C}_{\mathrm{n}} \mathrm{BEC}$ & betaine chloride alkyl esters $(n=10,12,14,16)$ & $18,5.5,1.9,0.33[17]$ \\
DMePC & $N$-dodecyl- $N$-methylpiperidinium chloride & $20[14]$ \\
DMeMC & $N$-dodecyl- $N$-methylmorpholinium chloride & $21[14]$ \\
C $\mathrm{n}$ APC & $N$-alkyl-pyridinium chlorides $(n=12,16,18)$ & $15,0.9,0.24[13]$ \\
1-nPiC & $N$-alkoxycarbonylmethyl- $N$ - & $118,21,5.5[17]$ \\
& $\quad \begin{array}{l}\text { alkyl-piperidinium } \\
\text { chlorides }(n=8,10,12)\end{array}$ & $220[18]$ \\
ONAC & $N$-octylnicotinamide chloride & $12,4[18]$ \\
DNAC & $N$-dodecylnicotinamide chloride & \\
\hline
\end{tabular}

charge distribution. Therefore, in order to minimize the influence of factors other then geometrical on $\mathrm{cmc}$ we took into account only cationic surfactants with chloride as counterion. However, the structures of the compounds are significantly different.

\section{Data}

The data set was chosen to contain only cationic surfactants, especially quaternary ammonium chloride salts. Literature data for $\mathrm{cmc}$ are given in Table 1. All values of $\mathrm{cmc}$ were measured in pure water at $25^{\circ} \mathrm{C}$.

The chemical structures of the surfactants taken into consideration and their abbreviations are shown in Fig. 1.

\section{Method}

\subsection{Connectivity indices $(\chi)$ and valence connectivity indices $\left(\chi^{\nu}\right)$}

The connectivity indices are topological descriptors of molecular structure based on a count of skeletal atom groupings. The molecular connectivity index is defined [11]:

$$
{ }^{m} \chi_{k}=\sum_{j=1}^{n_{m}} \prod_{i=1}^{m+1}\left(\delta_{i}\right)_{j}^{-0.5}
$$

where $m$ is the order of the connectivity index, $k$ denotes type of a fragment, which is divided into paths (P), clusters (C), and path/clusters (PC). In formula (1) $n_{m}$ is the number of the relevant paths, and $\delta_{i}$ is equal to the number of non-hydrogen atoms to which the $i$-th non-hydrogen atom is bonded. If $\delta_{i}$ is replaced by $\delta_{i}^{v}$, we obtain the expression for the $m$-th order valence connectivity index, ${ }^{m} \chi_{k}^{v}$, as follows: 
Fig. 1 Chemical structures of the investigated surfactants and their abbreviations.

$\mathrm{R}_{1}=\mathrm{C}_{\mathrm{n}} \mathrm{H}_{2 \mathrm{n}+1}$ ( $n=10,12,14,16)$,

$\mathrm{R}_{2}=\mathrm{C}_{\mathrm{n}} \mathrm{H}_{2 \mathrm{n}+1}(n=8,10,12)$,

$\mathrm{R}_{3}=\mathrm{C}_{\mathrm{n}} \mathrm{H}_{2 \mathrm{n}+1}$

( $n=10,12,14,16)$,

$\mathrm{R}_{4}=\mathrm{C}_{\mathrm{n}} \mathrm{H}_{2 \mathrm{n}+1}$

$(n=12,16,18)$,

$\mathrm{R}_{5}=\mathrm{C}_{\mathrm{n}} \mathrm{H}_{2 \mathrm{n}+1}(n=8,10,12)$,

$\mathrm{R}_{6}=\mathrm{C}_{\mathrm{n}} \mathrm{H}_{2 \mathrm{n}+1}(n=8,12)$
CnTAC<smiles>C[N+](C)(C)[P-]</smiles>

CnAC<smiles>[R2][NH3+]</smiles>

\section{CnBEC}<smiles>[R5]OC(=O)C[N+](C)(C)C</smiles>

\section{DMePC}<smiles>CCC[N+]1(C)CCCCC1</smiles>

DDAC<smiles>CC[N+](C)(C)C</smiles>

\section{DMeMC}

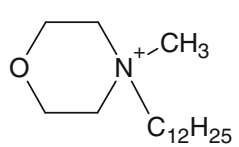

$\mathrm{Cl}^{-}$

\section{CnAPC}<smiles>[R4][n+]1ccccc1</smiles>

$\mathrm{Cl}^{-}$

1-nPiC<smiles>[R5]OC(=O)C[N+]1(C)CCCCC1</smiles>

$\mathrm{Cl}^{-}$

\section{ONAC \\ DNAC}<smiles>[R6][n+]1cccc(C(N)=O)c1</smiles> 
where $\delta_{i}^{v}$ is defined by

$$
\delta^{\nu}=\frac{Z^{v}-h}{Z-Z^{v}-1}
$$

where $Z^{v}, Z$ and $h$ are the number of valence electrons, the total number of electrons in the $i$-th atom, and the number of hydrogen atoms, respectively.

\subsection{Correlation formula}

The formula expressing the relationship between the $\log c m c$ and connectivity indices was generated using the least squares method. The statistical calculations were performed using the program STATISTICA 8.0 [19]. The final models were generated using the methodology described in previous papers $[4,7,12]$. In the process of searching for the equations three criteria were taken into account: a correlation coefficient $(r)$, a Fisher ratio value $(F)$ and a standard error $(s)$. The best relationship is that which has possibly highest values of $r$ and $F$, and simultaneously the lowest value of $s$.

\section{Results and discussions}

In the process of searching for the relationships between $\mathrm{cmc}$ and topological descriptors we used, just as in the previous paper [12], ten indices: five connectivity indices (from zeroth to fourth order) and five valence connectivity indices (from zeroth to fourth order). These indices were calculated for the compounds studied using Eqs. 1-3. The calculated connectivity indices are listed in Table 2.

Using a stepwise method we have obtained three models. In each model we start our correlation procedure with one index, i.e. the first step is common for each model and it is presented in Table 3.

We see that the best correlations in the first step are for the relationships containing the first-order valence connectivity index ${ }^{1} \chi^{\nu}$ and the zeroth-order valence connectivity index ${ }^{0} \chi^{\nu}$. These indices define first steps for Model 1 and Models 2 and 3, respectively.

In the first model the search for the best equation consists of three steps including the first step described above. The result of all correlations is presented in Table 4.

The best correlation in the first step in Model 1 is for the relationship containing the index ${ }^{1} \chi^{\nu}$, and we get the following formula:

$$
\log c m c=1.339-0.402 \cdot{ }^{1} \chi^{v}
$$

Next to this index we added the remaining indices separately. The result of the second step is presented in Table 4. Now we see that we get best correlation for the relationship containing in addition to the previous step the index ${ }^{4} \chi_{p c}$ (fourth-order connectivity index). The obtained formula for this step is as follows:

$$
\log c m c=1.623-0.531 \cdot{ }^{1} \chi^{v}+0.920 \cdot{ }^{4} \chi_{p c}
$$


Table 2 The connectivity indices and the experimental $\log c m c$ values

\begin{tabular}{|c|c|c|c|c|c|c|c|c|c|c|c|}
\hline & ${ }^{0} \chi$ & ${ }^{1} \chi$ & ${ }^{2} \chi$ & ${ }^{3} \chi_{c}$ & ${ }^{4} \chi_{p c}$ & ${ }^{0} \chi^{v}$ & ${ }^{1} \chi^{v}$ & ${ }^{2} \chi^{v}$ & ${ }^{3} \chi_{c}^{v}$ & ${ }^{4} \chi_{p c}^{v}$ & $\log C M C$ \\
\hline & 13.814 & 9.268 & 7.303 & 0.927 & 1.436 & 13.814 & 9.268 & 7.303 & 0.927 & 1.436 & \\
\hline & 13.814 & 9.268 & 7.303 & 0.927 & 1.436 & & & 6.855 & 0.927 & 1.436 & \\
\hline${ }_{10} \mathrm{BEC}$ & 13.855 & 8.454 & 7.487 & 1.849 & 0.961 & 12.887 & 7.611 & 6.433 & 1.620 & 0.601 & -1.745 \\
\hline $\mathrm{C}_{12} \mathrm{BEC}$ & 15.270 & 9.454 & 8.194 & 1.849 & 0.961 & 14.301 & 8.611 & 7.140 & 1.620 & 0.601 & -2.260 \\
\hline${ }_{14} \mathrm{BEC}$ & 16.684 & 10.454 & 8.901 & 1.849 & 0.961 & 15.715 & 9.611 & 7.847 & 1.620 & 0.601 & -2.721 \\
\hline${ }_{16} \mathrm{BEC}$ & 18.098 & 11.454 & 9.608 & 1.849 & 0.961 & 17.129 & 10.611 & 8.554 & 1.620 & 0.601 & -3.481 \\
\hline${ }_{12} \mathrm{APC}$ & 12.891 & .932 & 6.475 & 0.204 & 0.433 & 12.165 & 7.971 & 5.417 & 0.118 & 0.219 & -1.824 \\
\hline${ }_{16} \mathrm{APC}$ & 15.719 & 10.932 & 7.889 & 0.204 & 0.433 & 14.993 & 9.971 & 6.831 & 0.118 & 0.219 & -3.046 \\
\hline${ }_{18} \mathrm{APC}$ & 17.133 & 11.932 & 8.596 & 0.204 & 0.433 & 16.407 & 10.971 & 7.538 & 0.118 & 0.219 & -3.620 \\
\hline${ }_{10} \mathrm{TAC}$ & 10.864 & 6.561 & 5.786 & 1.561 & 0.75 & 10.864 & 6.561 & 5.786 & 1.561 & 0.75 & -1.215 \\
\hline $\mathrm{C}_{12} \mathrm{TAC}$ & 12.278 & 7.561 & 6.493 & 1.561 & 0.75 & 12.278 & 7.561 & 6.493 & 1.561 & 0.75 & -1.678 \\
\hline $14 \mathrm{TAC}$ & 13.692 & 8.561 & 7.200 & 1.561 & 0.75 & 13.692 & 8.561 & 7.200 & 1.561 & 0.75 & -2.347 \\
\hline $\mathrm{C}_{16} \mathrm{TAC}$ & 15.106 & 9.561 & 7.907 & 1.561 & 0.75 & 15.106 & 9.561 & 7.907 & 1.561 & 0.75 & -2.854 \\
\hline $\mathrm{C}_{18} \mathrm{TAC}$ & 16.521 & 10.561 & 8.614 & 1.561 & 0.75 & 16.521 & 10.561 & 8.614 & 1.561 & 0.75 & -3.461 \\
\hline 1-8PiC & 13.977 & 9.162 & 7.591 & 1.215 & 1.696 & 13.009 & 8.318 & 6.536 & 0.986 & 1.367 & -0.928 \\
\hline 1-10PiC & 15.391 & 10.162 & 8.298 & 1.215 & 1.696 & 14.423 & & 7.243 & 0.986 & 1.367 & \\
\hline 1-12PiC & 16.805 & 11.162 & 9.005 & 1.215 & 1.696 & 15.837 & 10.318 & 7.950 & 0.986 & 1.367 & -2.260 \\
\hline DDAC & 11.355 & 7.270 & 5.364 & 0.408 & 0.289 & 11.355 & 7.270 & 5.364 & 0.408 & 0.289 & -1.801 \\
\hline $\mathrm{C}_{8} \mathrm{AC}$ & 6.9498 & 4.414 & 2.768 & 0 & 0 & 6.9498 & 4.414 & 2.768 & 0 & 0 & -0.699 \\
\hline $\mathrm{C}_{10} \mathrm{AC}$ & 8.364 & 5.414 & 3.475 & 0 & 0 & 8.364 & 5.414 & 3.475 & 0 & 0 & -1.319 \\
\hline & 9.778 & 6.414 & 4.182 & 0 & 0 & 9.778 & 6.414 & 4.182 & 0 & 0 & -1.870 \\
\hline ONAC & 12.510 & 8.236 & 6.593 & 0.704 & 1.425 & 10.745 & 6.625 & 4.582 & 0.26 & 0.45 & -0.658 \\
\hline DNAC & 15.338 & 10.236 & 8.007 & 0.704 & 1.425 & 13.573 & 8.625 & 5.996 & 0.26 & 0.45 & -1.906 \\
\hline
\end{tabular}

Table 3 The values of the correlation coefficients for first step

\begin{tabular}{lcccccccccc}
\hline Connectivity index & ${ }^{0} \chi$ & ${ }^{1} \chi$ & ${ }^{2} \chi$ & ${ }^{3} \chi_{c}$ & ${ }^{4} \chi_{p c}$ & ${ }^{0} \chi^{\nu}$ & ${ }^{1} \chi^{\nu}$ & ${ }^{2} \chi^{v}$ & ${ }^{3} \chi_{c}^{v}$ & ${ }^{4} \chi_{p c}^{v}$ \\
\hline Correlation coefficient & 0.744 & 0.731 & 0.654 & 0.255 & 0.118 & $\mathbf{0 . 8 1 2}$ & $\mathbf{0 . 8 1 5}$ & 0.736 & 0.286 & 0.068 \\
\hline
\end{tabular}

Table 4 The values of the correlation coefficients for each step in the Model 1

\begin{tabular}{lcccccccccc}
\hline Connectivity index & ${ }^{0} \chi$ & ${ }^{1} \chi$ & ${ }^{2} \chi$ & ${ }^{3} \chi_{c}$ & ${ }^{4} \chi_{p c}$ & ${ }^{0} \chi^{v}$ & ${ }^{1} \chi^{v}$ & ${ }^{2} \chi^{v}$ & ${ }^{3} \chi_{c}^{v}$ & ${ }^{4} \chi_{p c}^{v}$ \\
\hline STEP 1 & 0.744 & 0.731 & 0.654 & 0.255 & 0.118 & 0.812 & $\mathbf{0 . 8 1 5}$ & 0.736 & 0.286 & 0.068 \\
STEP 2 & 0.826 & 0.842 & 0.858 & 0.820 & $\mathbf{0 . 9 7 4}$ & 0.817 & & 0.818 & 0.793 & 0.945 \\
STEP 3 & 0.983 & 0.974 & 0.983 & 0.983 & & $\mathbf{0 . 9 8 7}$ & & 0.978 & 0.980 & 0.977 \\
\hline
\end{tabular}




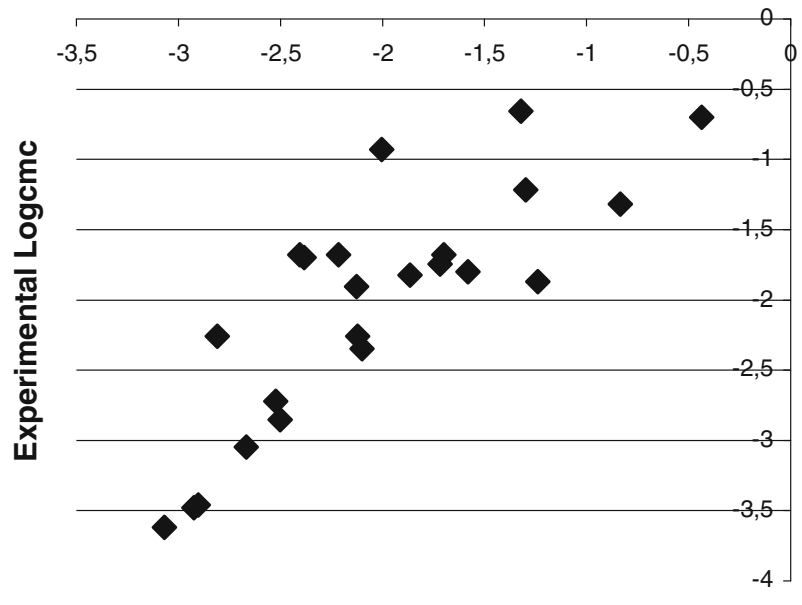

Calculated Logcmc

Fig. 2 Scatter plot of the calculated $\log c m c$ versus the experimental $\log c m c(r=0.815, F=41.59$, $s=0.5$ ). Step 1

In the third step the ${ }^{0} \chi^{v}$ index (zeroth-order valence connectivity index) was added (see Table 4) and the corresponding equation is the following:

$$
\log c m c=1.820-0.083 \cdot{ }^{1} \chi^{\nu}+0.977 \cdot{ }^{4} \chi_{p c}-0.304 \cdot{ }^{0} \chi^{v}
$$

At this step the process of searching for the best relationship was ended because the further additions of other indices did not change the correlation coefficient significantly. 3,4 .

The process of selecting the best relationship for Model 1 is illustrated in Figs. 2,

When we choose in the first step the zeroth-order valence connectivity index ${ }^{0} \chi^{\nu}$, we will obtain Model 2 and Model 3. The second step in these models is common and it is presented in Table 5.

Now we can see that the best correlations in the second step are for the relationships containing the fourth-order connectivity index ${ }^{4} \chi_{p c}$ (Model 2) and the second-order connectivity index ${ }^{2} \chi$ or fourth-order valence connectivity index ${ }^{4} \chi_{p c}^{v}$ (Model 3).

In second model the search for the best equation consists of two steps including the steps presented in Tables 3 and 5. The result of these correlations is presented in Table 6.

The obtained formula for the first step in Model 2 is:

$$
\log c m c=1.427-0.262 \cdot{ }^{0} \chi^{v}
$$

In the second step the best correlation is for the relationship containing, in addition to the previous step, the ${ }^{4} \chi_{p c}$ index and the obtained formula for that step is: 


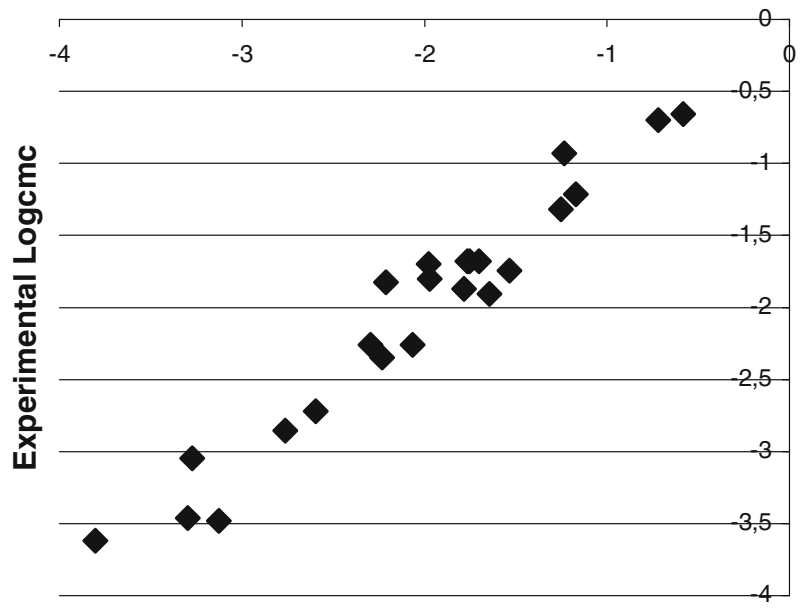

Calculated Logcmc

Fig. 3 Scatter plot of the calculated $\log c m c$ versus the experimental $\log c m c \quad(r=0.974$, $F=389.01, s=0.196)$. Step 2

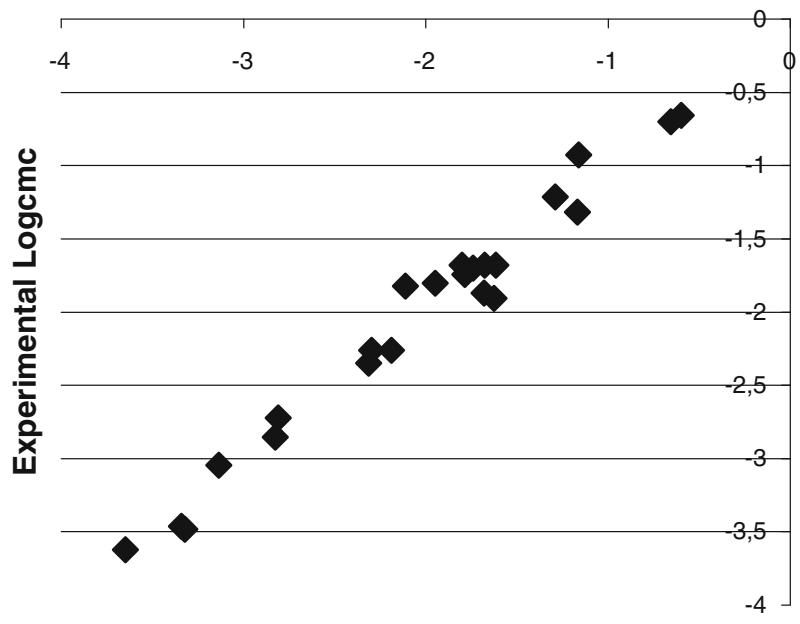

Calculated Logemc

Fig. 4 Scatter plot of the calculated $\log c m c$ versus the experimental $\log c m c(r=0.987$, $F=818.46, s=0.137$ ). Step 3 (final)

Table 5 The values of the correlation coefficients for second step in Model 2 and Model 3

\begin{tabular}{llllllllll}
\hline Connectivity index & ${ }^{0} \chi$ & ${ }^{1} \chi$ & ${ }^{2} \chi$ & ${ }^{3} \chi_{c}$ & ${ }^{4} \chi_{p c}$ & ${ }^{1} \chi^{v}$ & ${ }^{2} \chi^{v}$ & ${ }^{3} \chi_{c}^{v}$ & ${ }^{4} \chi_{p c}^{v}$ \\
\hline Correlation coefficient & 0.856 & 0.826 & $\mathbf{0 . 9 4 1}$ & 0.842 & $\mathbf{0 . 9 8 7}$ & 0.817 & 0.833 & 0.826 & $\mathbf{0 . 9 4 2}$ \\
\hline
\end{tabular}


Table 6 The values of the correlation coefficients for the steps in the Model 2

\begin{tabular}{lcccccccccc}
\hline Connectivity index & ${ }^{0} \chi$ & ${ }^{1} \chi$ & ${ }^{2} \chi$ & ${ }^{3} \chi_{c}$ & ${ }^{4} \chi_{p c}$ & ${ }^{0} \chi^{v}$ & ${ }^{1} \chi^{v}$ & ${ }^{2} \chi^{v}$ & ${ }^{3} \chi_{c}^{v}$ & ${ }^{4} \chi_{p c}^{v}$ \\
\hline STEP 1 & 0.744 & 0.731 & 0.654 & 0.255 & 0.118 & $\mathbf{0 . 8 1 2}$ & 0.815 & 0.736 & 0.286 & 0.068 \\
STEP 2 & 0.856 & 0.826 & 0.941 & 0.842 & $\mathbf{0 . 9 8 7}$ & & 0.817 & 0.833 & 0.826 & 0.942 \\
\hline
\end{tabular}

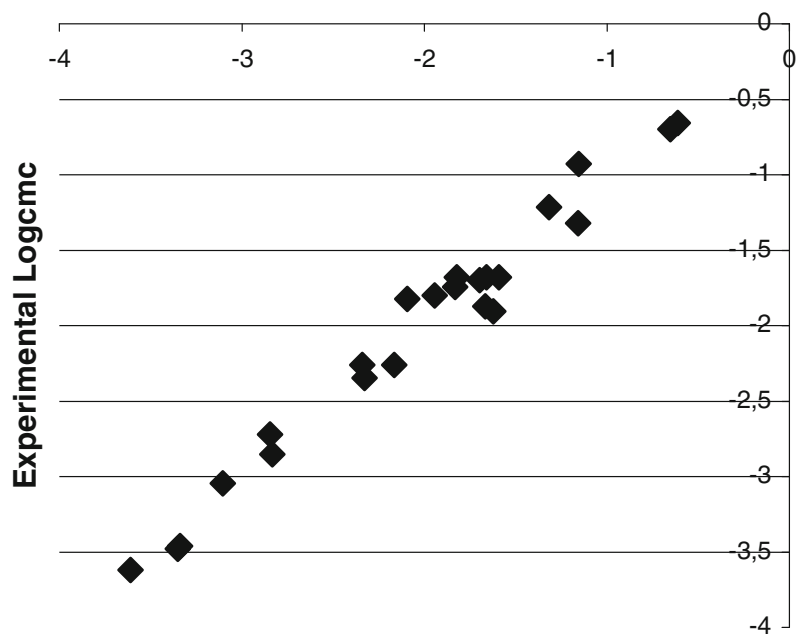

Calculated Logcmc

Fig. 5 Scatter plot of the calculated $\log c m c$ versus the experimental $\log c m c \quad(r=0.987$, $F=787.82, s=0.14$ ). Step 2 (final)

Table 7 The values of the correlation coefficients for each step in the Model 3

\begin{tabular}{lcccccccccc}
\hline Connectivity index & ${ }^{0} \chi$ & ${ }^{1} \chi$ & ${ }^{2} \chi$ & ${ }^{3} \chi_{c}$ & ${ }^{4} \chi_{p c}$ & ${ }^{0} \chi^{v}$ & ${ }^{1} \chi^{v}$ & ${ }^{2} \chi^{v}$ & ${ }^{3} \chi_{c}^{v}$ & ${ }^{4} \chi_{p c}^{v}$ \\
\hline STEP 1 & 0.744 & 0.731 & 0.654 & 0.255 & 0.118 & $\mathbf{0 . 8 1 2}$ & 0.815 & 0.736 & 0.286 & 0.068 \\
STEP 2 & 0.856 & 0.826 & 0.941 & 0.842 & 0.987 & & 0.817 & 0.833 & 0.826 & $\mathbf{0 . 9 4 2}$ \\
STEP 3 & 0.990 & 0.965 & $\mathbf{0 . 9 9 3}$ & 0.943 & 0.988 & & 0.948 & 0.956 & 0.943 & \\
\hline
\end{tabular}

$$
\log c m c=1.832-0.358 \cdot{ }^{0} \chi^{v}+0.982 \cdot{ }^{4} \chi_{p c}
$$

The scatter plot of the logarithm of $c m c$ calculated from Eq. 8 versus the experimental one is shown in Fig. 5.

In Model 3 the search for the best relationship consists of three steps including the steps presented in Tables 3 and 5. The result of these correlations is presented in Table 7.

The result of the first step for Model 3 is presented in Table 7 and the formula for that step is described by Eq. 7. From this table in the second step we get best correlation for the relationship which, in addition to the first step, contains the index ${ }^{4} \chi_{p c}^{v}$ (fourth-order valence connectivity index). The obtained formula for this step is: 


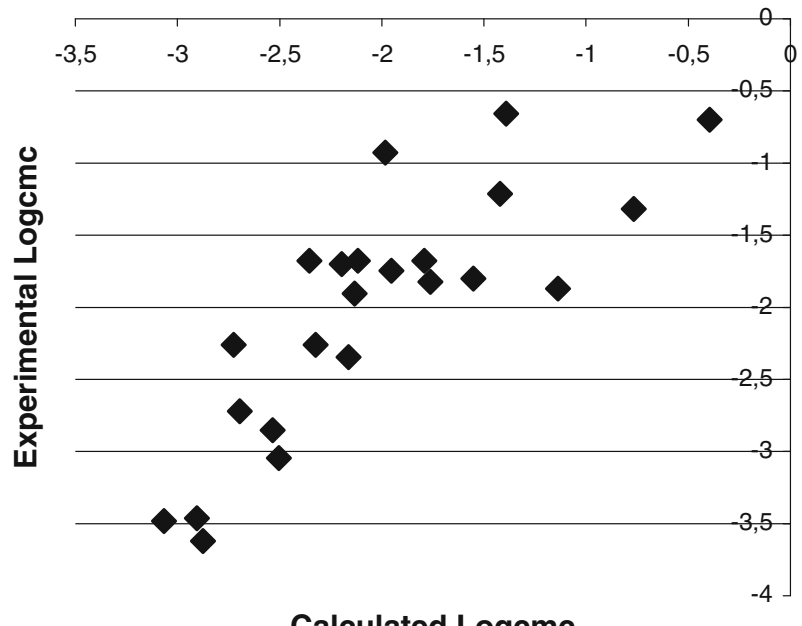

Fig. 6 Scatter plot of the calculated $\log c m c$ versus the experimental $\log c m c(r=0.812$, $F=40.614, s=0.5$ ). Step 1

$$
\log c m c=1.810-0.3391 \cdot{ }^{0} \chi^{\nu}+0.961 \cdot{ }^{4} \chi_{p c}^{\nu}
$$

The best correlation in third step in Model 3 is for the relationship which, in addition to the previous one, contains the index ${ }^{2} \chi$ (second-order connectivity index) and the formula for $\log c m c$ becomes:

$$
\log c m c=2.603-0.710 \cdot{ }^{0} \chi^{\nu}+0.694 \cdot{ }^{4} \chi_{p c}^{v}+0.604 \cdot{ }^{2} \chi
$$

The process of selecting the best relationship for Model 3 is illustrated in Figs. 6, 78.

It is worth noting that Model 2 and Model 3 show the best correlations among all the formulae containing two and three indices respectively.

The specification of all models is listed in Table 8 .

The calculated logarithm of $c m c$ values using the Models 1-3 and experimental $\log c m c$ for the surfractants studied are listed in Table 9.

From the previous calculation and from Table 9 it follows that Model 3 is the best, and the calculated values of $\log c m c$ are very close to the experimental ones. The structures of the compounds used for the correlation are diverse, which allows suggesting that the obtained equation for the prediction of $\log \mathrm{cmc}$ from only topological indices may have quite a general meaning.

It is worth noting that all the models contain ${ }^{0} \chi^{\nu}$ index with negative coefficients and ${ }^{4} \chi_{p c}$ or ${ }^{4} \chi_{p c}^{\nu}$ with positive ones. Thus, as ${ }^{0} \chi^{\nu}$ increases the $c m c$ decreases, and in the case of ${ }^{4} \chi_{p c}$ or ${ }^{4} \chi_{p c}^{v}$ it is vice versa. This may suggest that the index ${ }^{0} \chi^{\nu}$ includes some information about hydrophobicity, while ${ }^{4} \chi_{p c}$ and ${ }^{4} \chi_{p c}^{v}$ about hydrophilicity of the cationic surfactants studied. 


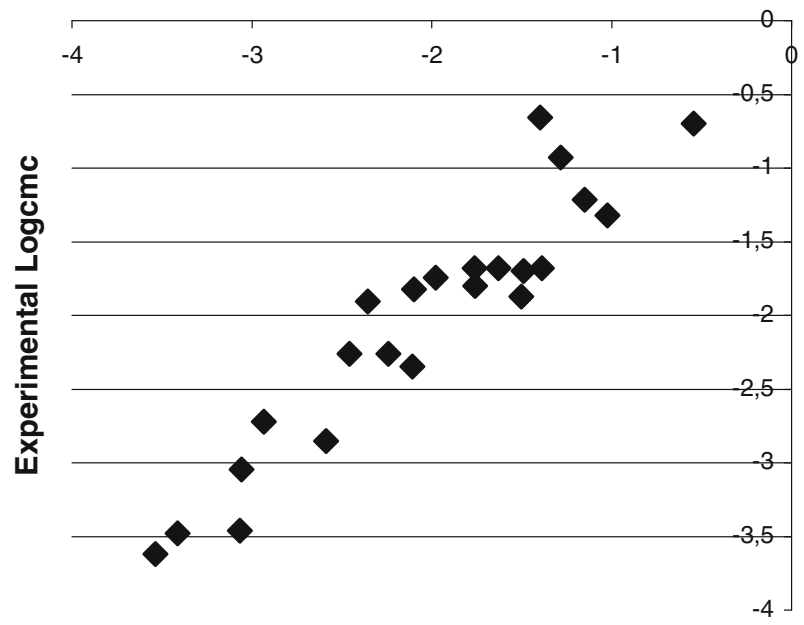

Calculated Logcmc

Fig. 7 Scatter plot of the calculated $\log c m c$ versus the experimental $\log c m c \quad(r=0.942$, $F=165.895, s=0.29$ ). Step 2

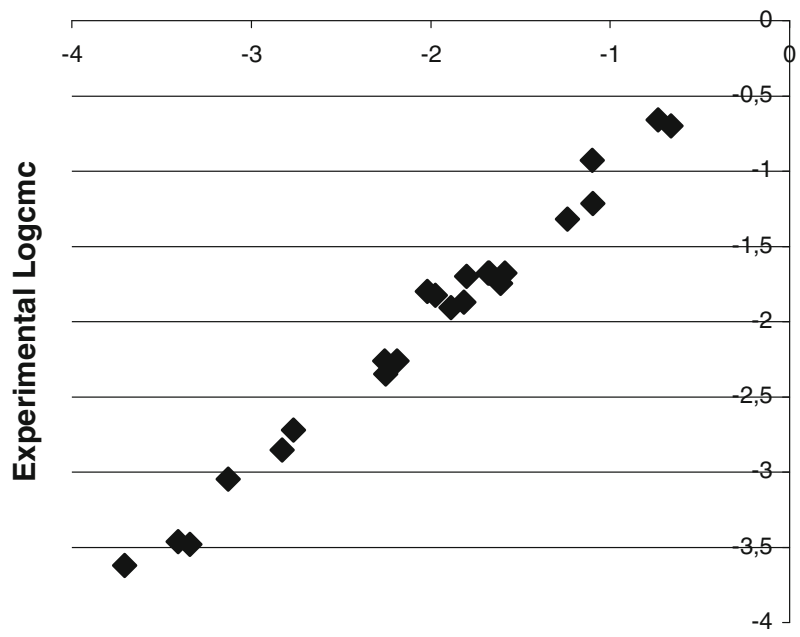

Calculated Logcmc

Fig. 8 Scatter plot of the calculated $\log c m c$ versus the experimental $\log c m c(r=0.993$, $F=1477.17, s=0.102$ ). Step 3 (final)

Table 8 Specification of Models 1-3

$$
\begin{array}{ll}
\text { ModelRelationship } \\
\hline 1 & \log c m c=1.820-0.083 \cdot{ }^{1} \chi^{v}+0.977 \cdot{ }^{4} \chi_{p c}-0.304 \cdot{ }^{0} \chi^{v} \\
2 & \log c m c=1.832-0.358 \cdot{ }^{0} \chi^{v}+0.982 \cdot{ }^{4} \chi_{p c} \\
3 & \log c m c=2.603-0.710 \cdot{ }^{0} \chi^{v}+0.694 \cdot{ }^{4} \chi_{p c}^{v}+0.604 \cdot{ }^{2} \chi \\
\hline
\end{array}
$$


Table 9 Calculated and literature values of $\log c m c$ for studied 23 cationic surfactants

\begin{tabular}{|c|c|c|c|c|}
\hline \multirow[t]{2}{*}{ Compound } & \multirow[t]{2}{*}{ Experimental $\log c m c$} & \multicolumn{3}{|c|}{ Calculated $\log c m c$} \\
\hline & & Model 1 & Model 2 & Model 3 \\
\hline DMePC & -1.699 & -1.740 & -1.698 & -1.799 \\
\hline DMeMC & -1.678 & -1.615 & -1.591 & -1.587 \\
\hline $\mathrm{C}_{10} \mathrm{BEC}$ & -1.745 & -1.786 & -1.833 & -1.61 \\
\hline $\mathrm{C}_{12} \mathrm{BEC}$ & -2.260 & -2.298 & -2.339 & -2.187 \\
\hline $\mathrm{C}_{14} \mathrm{BEC}$ & -2.721 & -2.810 & -2.845 & -2.764 \\
\hline $\mathrm{C}_{16} \mathrm{BEC}$ & -3.481 & -3.322 & -3.351 & -3.341 \\
\hline $\mathrm{C}_{12} \mathrm{APC}$ & -1.824 & -2.112 & -2.094 & -1.974 \\
\hline $\mathrm{C}_{16} \mathrm{APC}$ & -3.046 & -3.136 & -3.105 & -3.128 \\
\hline $\mathrm{C}_{18} \mathrm{APC}$ & -3.620 & -3.649 & -3.611 & -3.705 \\
\hline $\mathrm{C}_{10} \mathrm{TAC}$ & -1.215 & -1.290 & -1.317 & -1.097 \\
\hline $\mathrm{C}_{12} \mathrm{TAC}$ & -1.678 & -1.803 & -1.823 & -1.674 \\
\hline $\mathrm{C}_{14} \mathrm{TAC}$ & -2.347 & -2.315 & -2.328 & -2.251 \\
\hline $\mathrm{C}_{16} \mathrm{TAC}$ & -2.854 & -2.827 & -2.834 & -2.829 \\
\hline $\mathrm{C}_{18} \mathrm{TAC}$ & -3.461 & -3.340 & -3.34 & -3.407 \\
\hline 1-8PiC & -0.928 & -1.163 & -1.155 & -1.101 \\
\hline 1-10PiC & -1.678 & -1.676 & -1.661 & -1.679 \\
\hline $1-12 \mathrm{PiC}$ & -2.260 & -2.188 & -2.166 & -2.256 \\
\hline DDAC & -1.801 & -1.948 & -1.945 & -2.021 \\
\hline $\mathrm{C}_{8} \mathrm{AC}$ & -0.699 & -0.656 & -0.653 & -0.661 \\
\hline $\mathrm{C}_{10} \mathrm{AC}$ & -1.319 & -1.168 & -1.159 & -1.238 \\
\hline $\mathrm{C}_{12} \mathrm{AC}$ & -1.870 & -1.681 & -1.665 & -1.816 \\
\hline ONAC & -0.658 & -0.600 & -0.611 & -0.733 \\
\hline DNAC & -1.906 & -1.625 & -1.623 & -1.888 \\
\hline
\end{tabular}

The obtained relationships were used to predict $\log \mathrm{cmc}$ for some cationic surfactants which were not used in the correlations. In Table 10 the predicted $\log c m c$ values were compared with the experimental ones.

The agreement between calculated and experimental $\log \mathrm{cmc}$ values seems to be quite good and is the best for Model 3. This confirms the usefulness of the obtained correlation for predicting $\log \mathrm{cmc}$ for cationic surfactants having chloride as counterion.

\section{Conclusion}

Relationships between molecular connectivity indices and critical micelle concentration $(\mathrm{cmc})$ of cationic surfactants, mainly quaternary ammonium chloride salts with one hydrophobic tail, were investigated using the quantitative structure-property relationship approach. Three models containing ${ }^{0} \chi^{\nu}$ and ${ }^{4} \chi_{p c}$ or ${ }^{4} \chi_{p c}^{v}$ indices were 
Table 10 Test Models 1-3

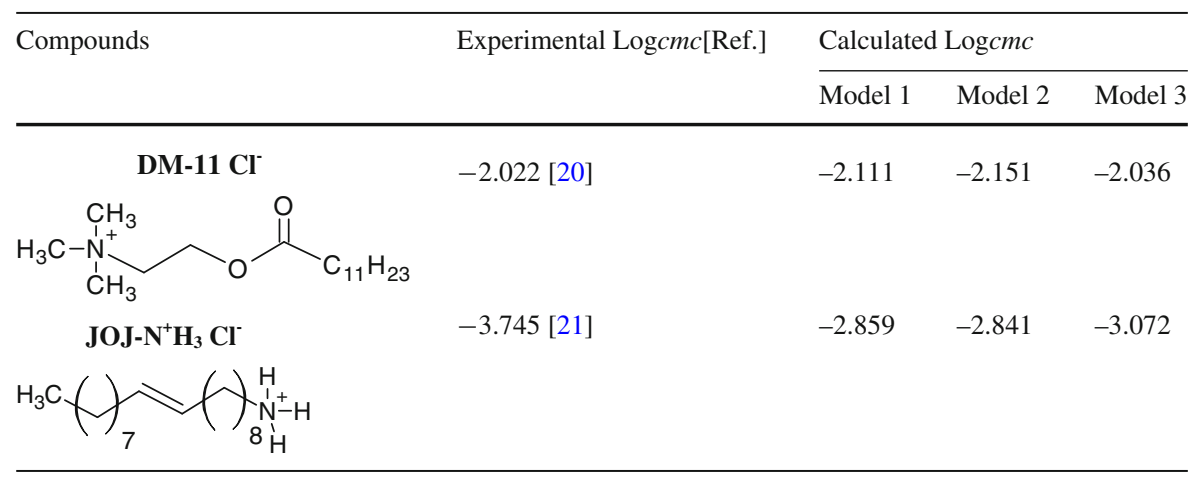

obtained. The ${ }^{0} \chi^{\nu}$ index is with negative while the ${ }^{4} \chi_{p c}$ and ${ }^{4} \chi_{p c}^{\nu}$ indices are with positive coefficients. It is suggested that the index ${ }^{0} \chi^{\nu}$ includes some information about hydrophobicity while indices ${ }^{4} \chi_{p c}$ and ${ }^{4} \chi_{p c}^{v}$ include some information about hydrophilicity of the cationic surfactants studied. The best model contains ${ }^{0} \chi^{\nu}$ and ${ }^{4} \chi_{p c}$ indeces and also ${ }^{2} \chi$ index. The obtained relationship should enable to predict the $c m c$ of cationic surfactants with chloride as counterion.

Open Access This article is distributed under the terms of the Creative Commons Attribution Noncommercial License which permits any noncommercial use, distribution, and reproduction in any medium, provided the original author(s) and source are credited.

\section{References}

1. H.B. Klevens, J. Am. Oil Chem. Soc. 30, 74-80 (1953)

2. P.D.T. Huibers, V.S. Lobanov, A.R. Katritzky, D.O. Shah, M. Karelson, Langmuir 12, 1462-1470 (1996)

3. Z. Wang, G. Li, X. Zhang, R. Wang, A. Lou, Colloids Surf. A 197, 37-45 (2002)

4. S. Yuan, Z. Cai, G. Xu, Y. Jiang, Colloid Polym Sci. 280, 630-636 (2002)

5. R.A. Saunders, J.A. Platts, J. Phys. Org. Chem. 17, 431-438 (2004)

6. P.D.T. Huibers, V.S. Lobanov, A.R. Katritzky, D.O. Shah, M. Karelson, J. Colloid Interface Sci. 187, 113-120 (1997)

7. S. Yuan, Z. Cai, G. Xu, Y. Jiang, J. Dispers. Sci. Technol. 23, 465-472 (2002)

8. M. Jalali-Heravi, E. Konouz, J. Surfact. Deterg. 3, 47-52 (2000)

9. M. Jalali-Heravi, E. Konouz, J. Surfact. Deterg. 6, 25-30 (2003)

10. N. Anoune, M. Nouiri, Y. Berrah, J.Y. Gauvrit, P. Lanteri, J. Surfact. Deterg. 5, 45-53 (2002)

11. L.B. Kier, L.H. Hall, Molecular Connectivity in Structure (Activity Analysis Research Studies Press LTD, Letchworth, 1986)

12. A. Mozrzymas, B. Różycka-Roszak, Combinatorial Chemistry \& High Throughput Screening 13, 39-44 (2010)

13. M.J. Rosen, Surfactants and Interfacial Phenomena (Wiley, New York, 1978)

14. B. Różycka-Roszak, J. Colloid Interface Sci. 140, 538-540 (1990)

15. F. Kopecky, T. Fazekas, B. Kopecka, P. Kaclik, Acta Facult. Pharm. Univ. Comenianae 54, 84-94 (2007)

16. R. de Lisi, E. Fisicaro, S. Milioto, J. Solution Chem. 17, 1015-1041 (1988)

17. B. Różycka-Roszak, P. Misiak, E. Woźniak, A. Mozrzymas, Z. Dega-Szafran, Colloids Surf. A 318, 301-306 (2008)

18. R. Lisi, E. Fisicaro, S. Milioto, E. Pelizzetti, P. Savarino, J. Solution Chem. 19, 247-270 (1990) 
19. StatSoft, Inc. (2008). STATISTICA (data analysis software system), version 8.0. www.statsoft.com

20. J. Łuczyński, E. Woźniak, S. Witek, B. Różycka-Roszak, The synthesis of $N$-methyl quaternary derivatives of 2-dimethylaminoethyl laurate and their micellization process, in Surfactants and Disperse Systems in Theory and Practice, Scientific Papers of the Institute of Organic and Polymer Technology of the Wroctaw University of Technology, vol. 48, Wrocław, 2000, pp. 113-116

21. S. Magdassi, A. Shani, JAOCS 67, 605-606 (1990) 